\author{
يحيى زارع مهرجردى * (استاد) \\ دانشكدهى مهندسى صنايع، دانشكاه يزد (ادر) \\ فهيمه فارغ (كارشناس ارشد) \\ دانشكدهى مهندسى صنايع، دانشكاه علم وهنر
}

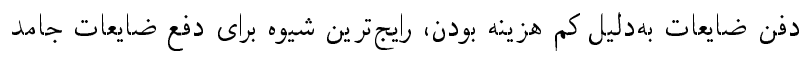

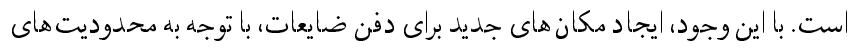

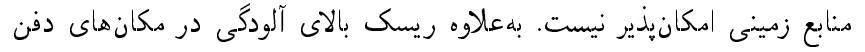

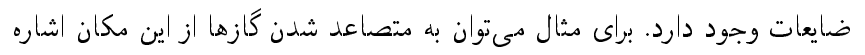

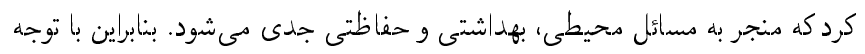

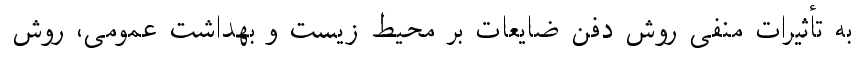

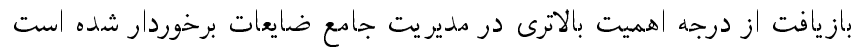

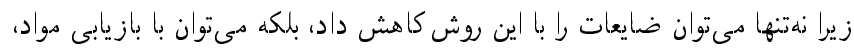

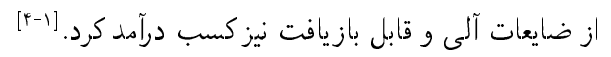

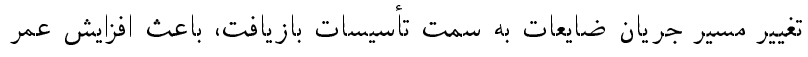

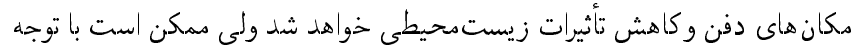

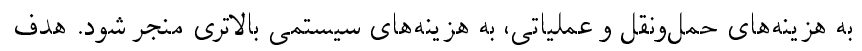

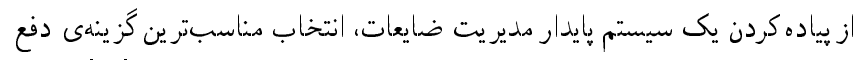

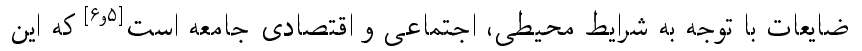

وازگًان كليدى: مديريت ضايعات جامد، شهر يزد، برنامهريزى كسرى، برنامه ريزى احتمالى، برنامهريزى كسرى خطى تصادفى.

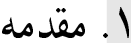

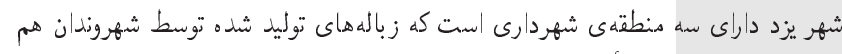

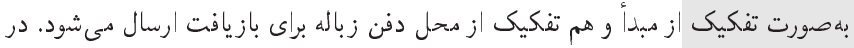

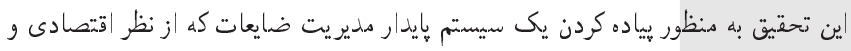

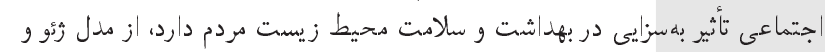

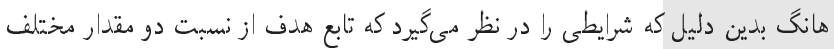

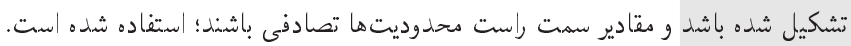

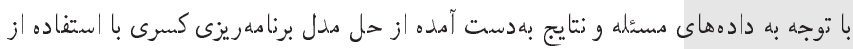

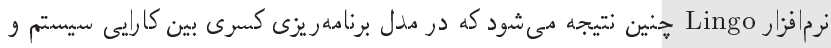

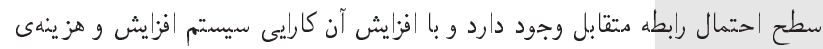

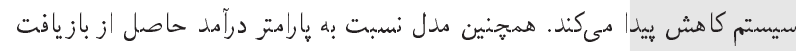

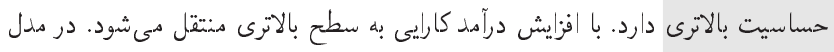

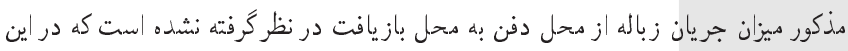
دقاله دحققين به آن يرداختهاند.

واثرى (ازباله)) معناهاى متفاوتى دارد و در حالت كلى به معناى ((نخواستن) است.

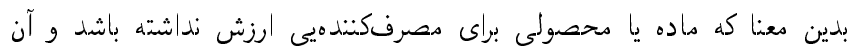

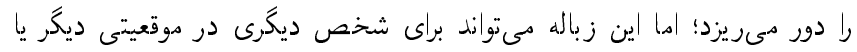

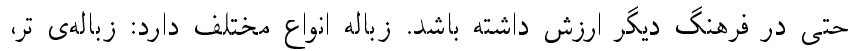

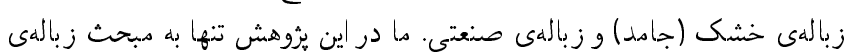

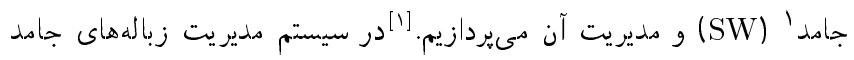

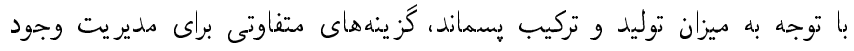

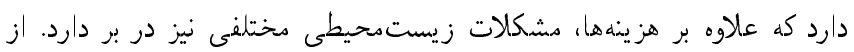

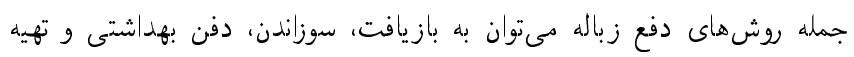

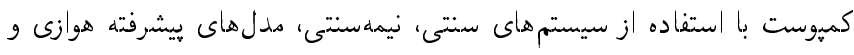

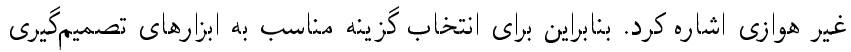

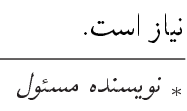

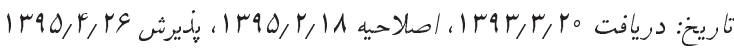




\section{r. متدولوزى}

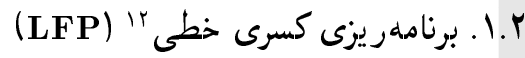

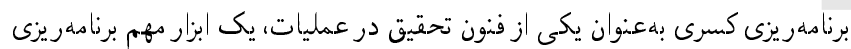

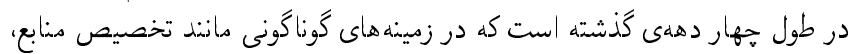

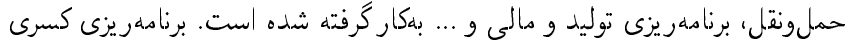

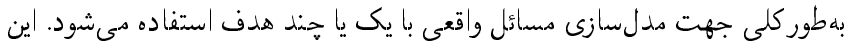

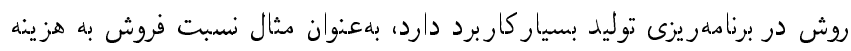

نمونهيى از اهداف كسرى در برنامهريزى توليد است.

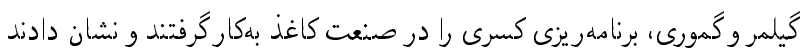

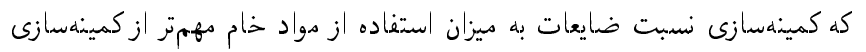

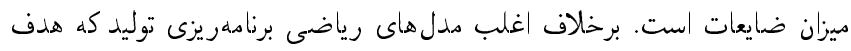

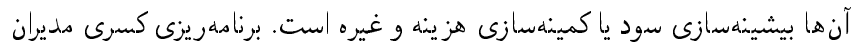

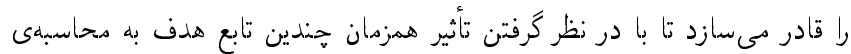
بهرهورى و كارايى سازمان بيردازند.

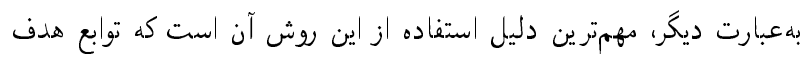

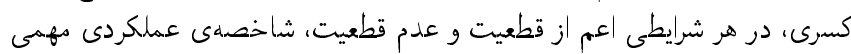

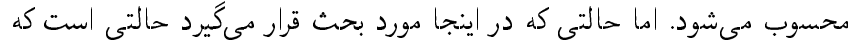
توابع صورت و مخرج، توابع خطى از متغيرهاى تصميم هستند كه ده آن آن را مسائل

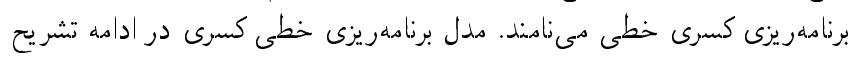

$\operatorname{Max}(\operatorname{Min}) \frac{C^{t} x+\alpha}{D^{t} x+\beta}$

s.t $\quad A x=b \quad x \geq$ 。

تالعاده، $C^{t}$ ترانهادهى اين بردارهاست، $A$ يك ماتريس

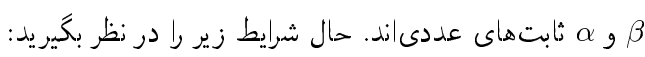

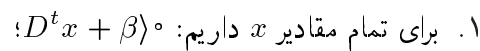
r. r. تابع هدف بهصورت ييوسته مشتقيذير است؛ r. فضاى جواب تهى است و همجنين كراندار باشد.

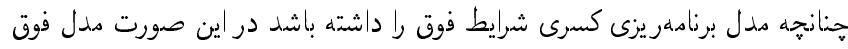

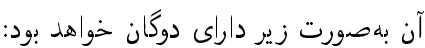

$\min g(y, z)=z$

$A^{t} y+D^{t} z \geq c^{t}$

$-B^{t} y+\beta z=\alpha$

$y \geq$ 。

كه در آن y يك بردار ستونى با mمؤلفه، ZZ يك متغير دقياس و نماد t نشاندهندهى

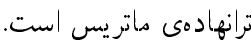

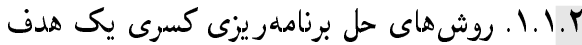

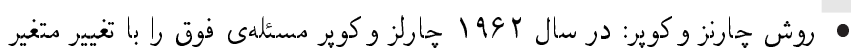

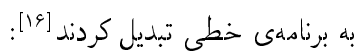

$T=\frac{1}{D^{t} x+\beta} \quad y=T x$
امر منجر به بهينه كردن سامانهى مديريت بسماند مىشود. مديريت إيدار يا مديريت

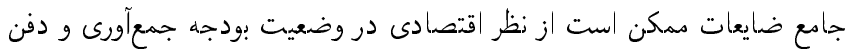

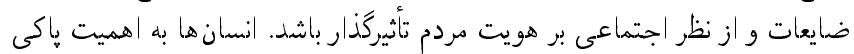

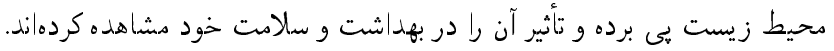

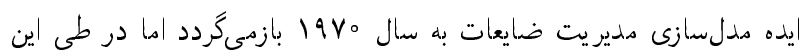

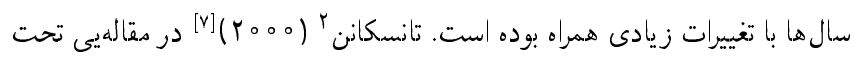

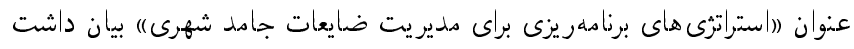

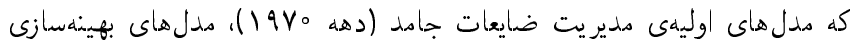

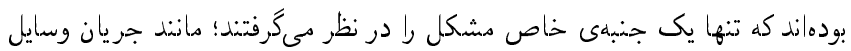

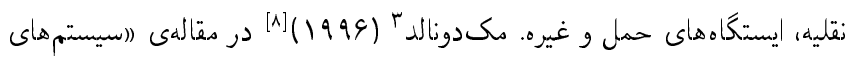

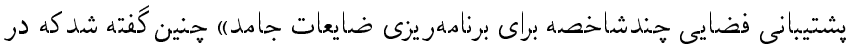

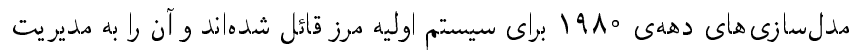

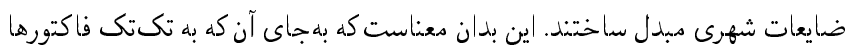

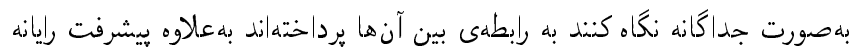

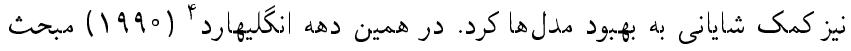

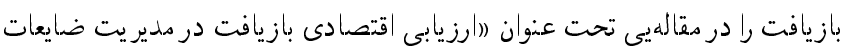

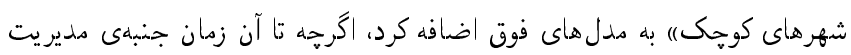

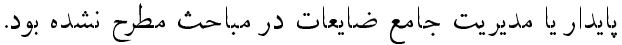

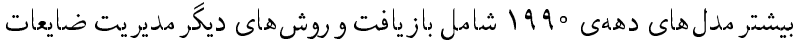

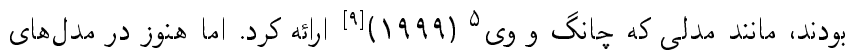

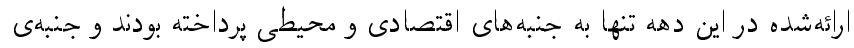

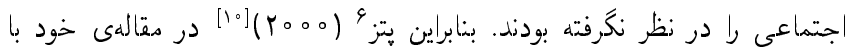

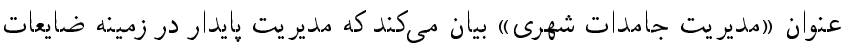

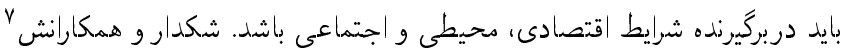

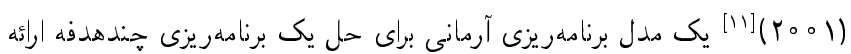

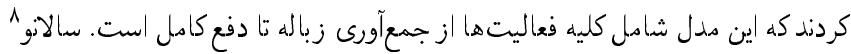

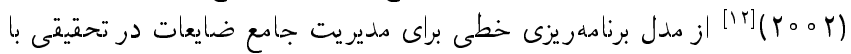

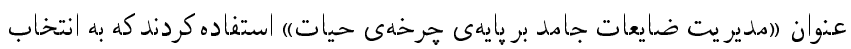

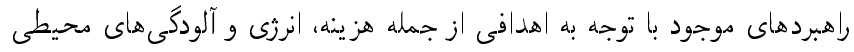

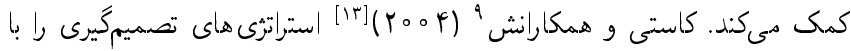
توجه به إرامترهاى دحيطى در زمينهى مديريت ضايعات جامد در در تحقيقى با عنوان

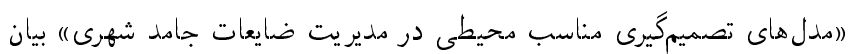

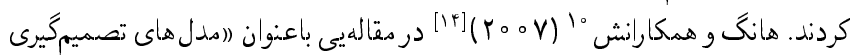

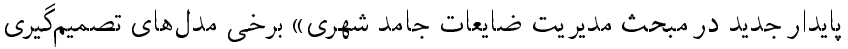

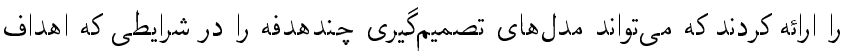

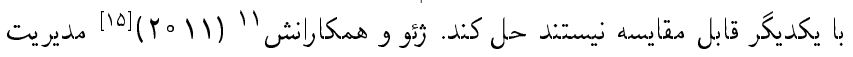

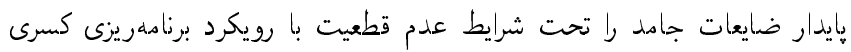

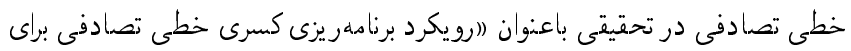

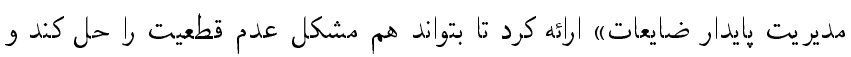

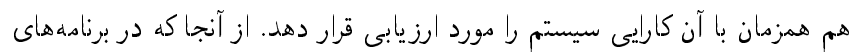

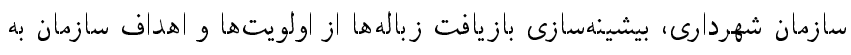

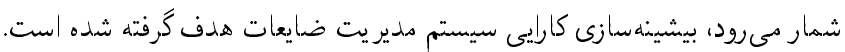

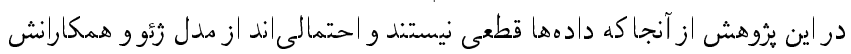

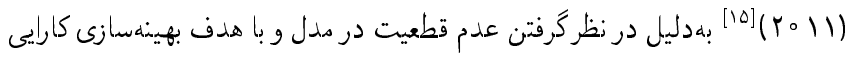
سيستم استفاده شده است 


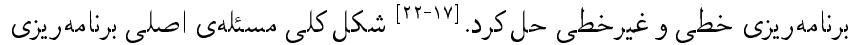

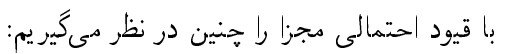

$\operatorname{Max} z \quad \sum_{j=1}^{n} c_{j} x_{j}$

S.t $P\left\{\sum a_{i j} x_{j} \leq b_{i}\right\} \geq \alpha_{i}$

$a_{i} \in[0,1] \quad x_{j}>\circ \quad i=1, \ldots, m$

كه قيد iام بايد با احتمال

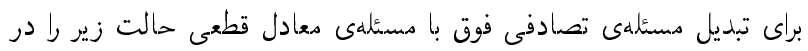

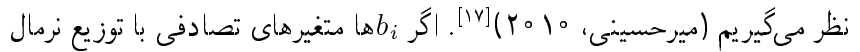
و ميانكين E

$P\left\{b_{i} \geq \sum a_{i j} x_{j}\right\} \geq \alpha_{i} \quad i=1, \ldots, m$

$p\left\{\frac{b_{i}-E\left(b_{i}\right)}{\sqrt{\operatorname{var}\left(b_{i}\right)}} \geq \frac{\sum a_{i j} x_{j}-E\left(b_{i}\right)}{\sqrt{\operatorname{var}\left(b_{i}\right)}}\right\} \geq a_{i} \quad i=1, \ldots, m$

عبارت فوق وقتى برقرار است كه داشته باشيم:

$\frac{\sum a_{i j} x_{j}-E\left(b_{i}\right)}{\operatorname{var}\left(b_{i}\right)} \leq K \alpha_{i} \quad i=1, \ldots, m$

بنابراين قيد احتمالى فوق معادل قيد خطى قطعى زير است:

$\sum a_{i j} x_{j} \leq E\left(b_{i}\right)+K \alpha_{i} \sqrt{\operatorname{var}\left(b_{i}\right)} \quad i=1, \ldots, m$

بنابراين در اين حالت، قيد احتمالى مسئله را مىتوان به يك قيد قطعى تبديل كرد، كه مدل برنامهريزى خطى هان ال از آن حاصل مئ آشود.

$\operatorname{Man} \sum_{j=1}^{n} c_{j} x_{j}$

S.T. $\sum_{j=1}^{n} a_{i j} x_{j} \leq E\left(b_{i}\right)+k \alpha_{i} \sqrt{\operatorname{var} b_{i}} \quad i=1, \ldots, m$

$x_{j} \geq \circ \quad a_{i} \in\left({ }^{\circ}, 1\right) \quad i=1, \ldots, m \quad j=1, \ldots, n$

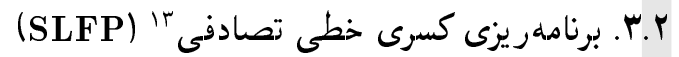

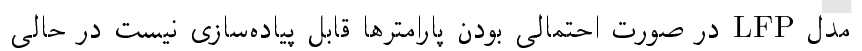

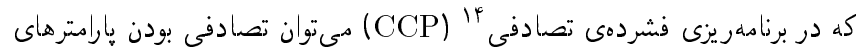

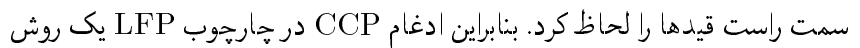

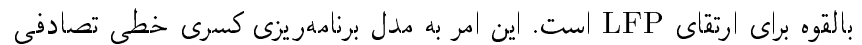
منجر مىشود. (SLFP)

$\operatorname{Max} f(x) \frac{C x+\alpha}{D x+\beta}$

$p_{r}\left[A_{i}(t) x \leq b_{i}(t)\right] \geq 1-p_{i, i=1, \mathrm{r}, \ldots, m}$

$x \geq$ 。

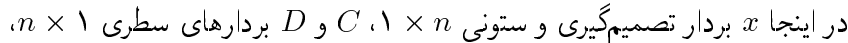

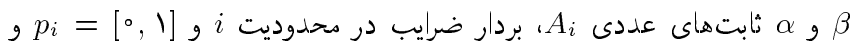

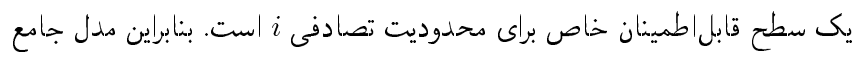

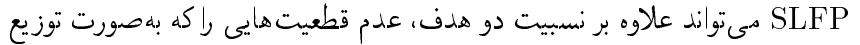
احتمالات شرح داده شدهاند، در مدلسازى لحاظ كند.
با اين تغيير مسئلى برنامهريزى خطى دطابق فرمول |f بهدست آمد. براى حالتى

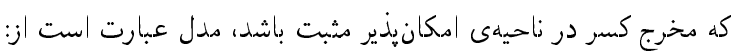

$\operatorname{Max}(\min ) C^{t} y+\alpha T$

s.t $\quad A y-b T={ }^{\circ}, D^{t} y+\beta T=1 \quad y>\circ, T>\circ$

و براى حالتى كه مخرج كسر در ناحيهى امكانيذير منفى باشد مدل عبارت خواهد

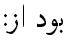

$\operatorname{Max}(\min )-C^{t} y-\alpha T$

s.t $\quad A y-b T={ }^{\circ}, D^{t} y+\beta T=-1 \quad y>\circ, T>\circ$

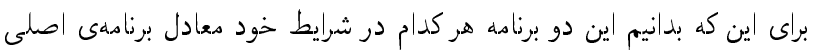

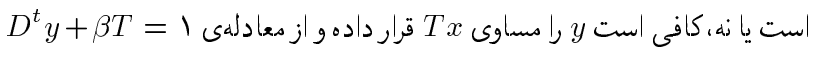

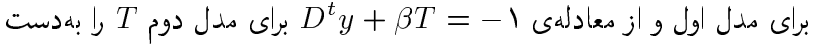

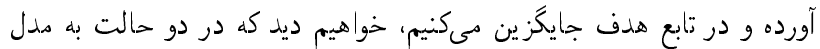

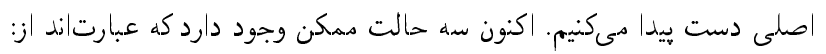
1. اكر مخرج در تمام ناحيهى امكانيذير مثبت باشد مدل اول را حل مىكنيم. r. اكر مخرج در تمام ناحيهى امكانيذير منفى باشد مدل دوم را حل مىكنيم.

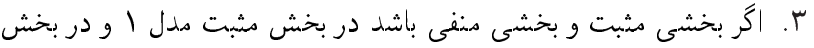

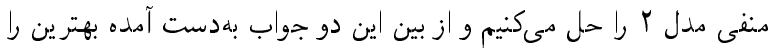

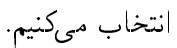

• الكَريتم كَيلمر وگمورى: در اين روش فرضم بر بر آن است كه S مجموعه امكانيذير

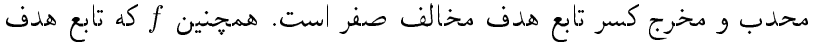

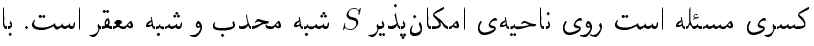

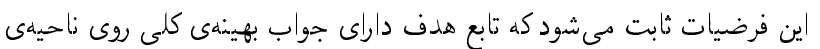

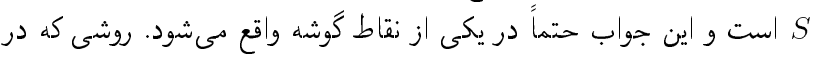

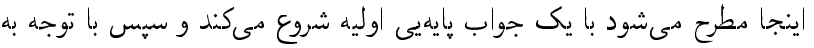

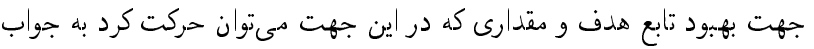

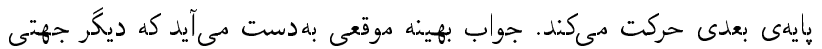

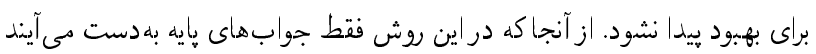

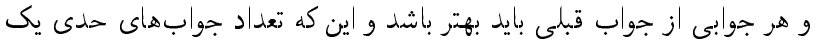

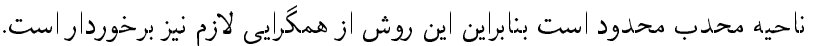

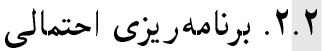

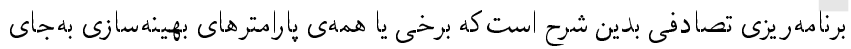

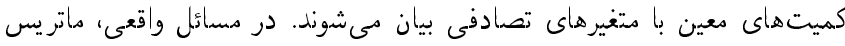

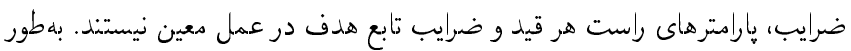

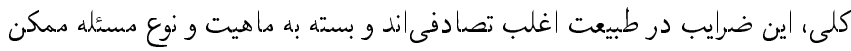

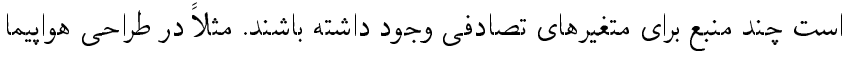

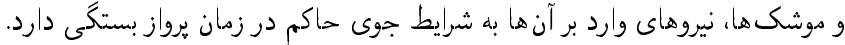

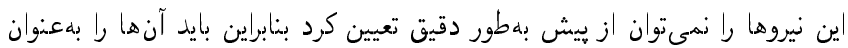

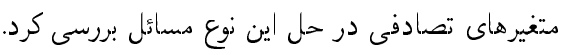

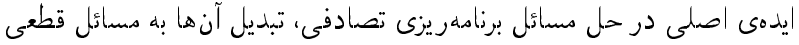

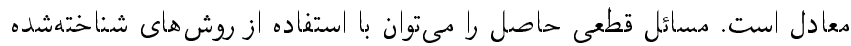




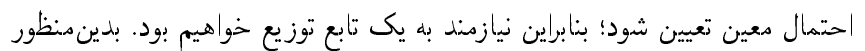

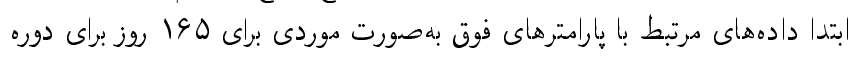

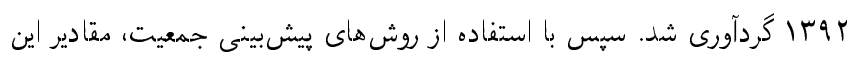

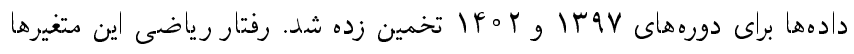

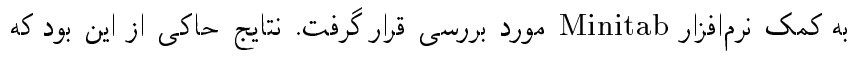

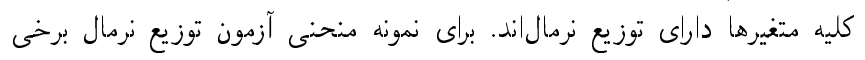

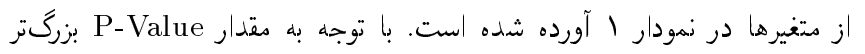

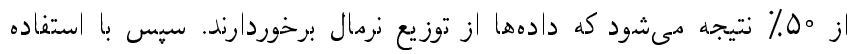

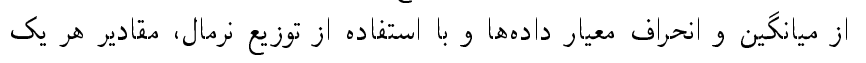

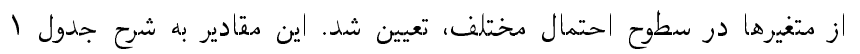

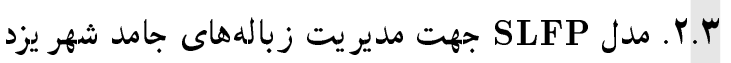

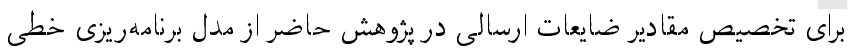

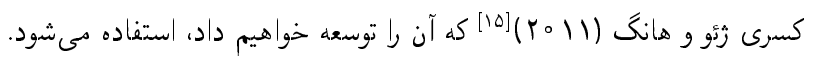

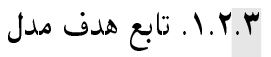

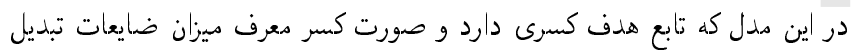

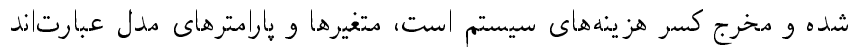
از: از مكان دفن؛ تأسيسات بازيافت؛

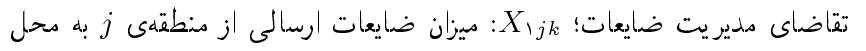
دفن در دوره k؛ ؛ ث محدوديت تقاضاى مديريت ضايعات. r. (بط. مالعهى موردى

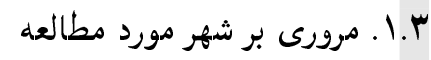

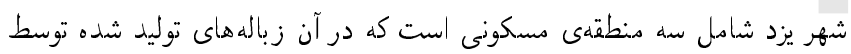

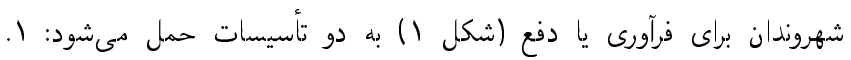

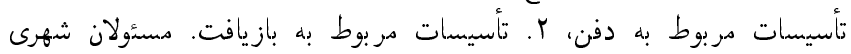

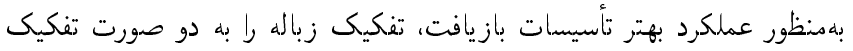

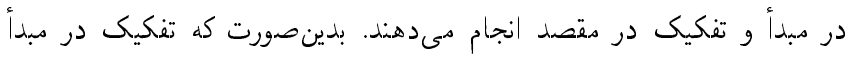

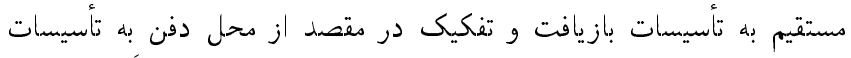

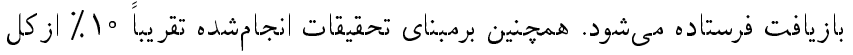

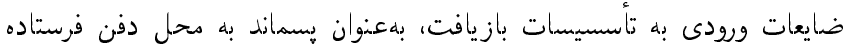

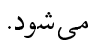

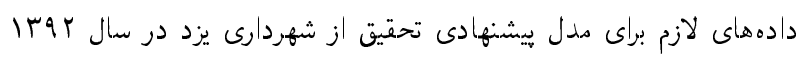

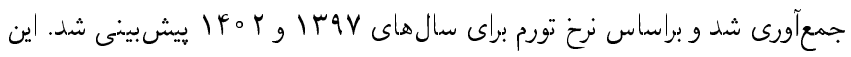

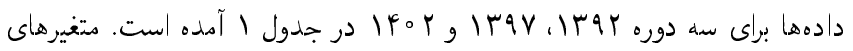

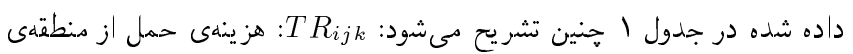

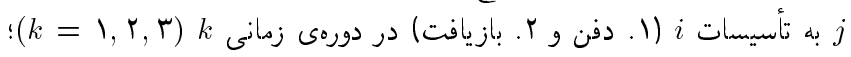
ملأ $O P_{\backslash k}$ مبدأ؛ بازيافت به دفن؛

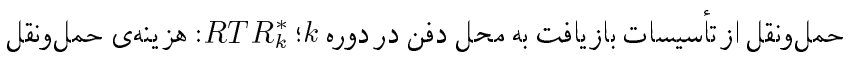
از تأسيسات دفن به محل بازيافت در دوره k؛

علاوه بر متغيرهاى فوق لازم است تا ظرفيت بازيافت، ظرفيت دفن و ميزان

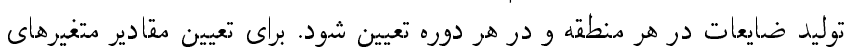

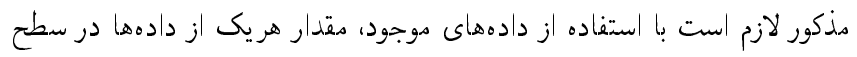

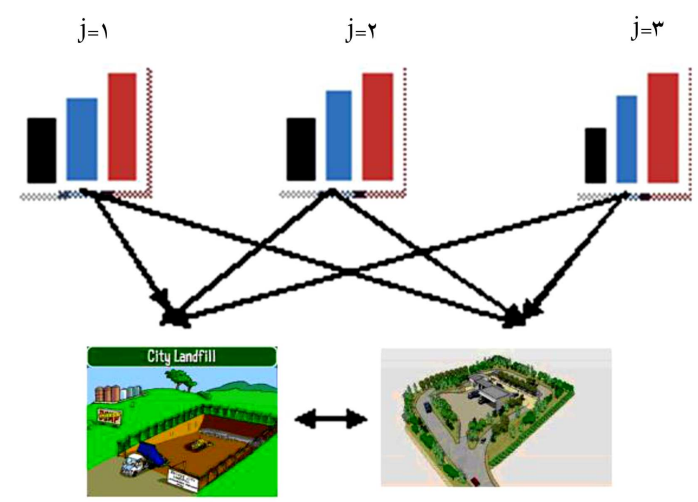

محل دفن

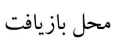
شكل ا. سيستم مورد مطالعه. نمودار I. توزيع نرمال ميزان كل دفن زبالههاى سال بوسبا. $\sum_{j=1}^{r} \sum_{k=1}^{r} L_{k} \cdot x_{r} k_{k}(1-R F)$ $\operatorname{Max} f=\frac{\sum_{j=1}^{r} \sum_{k=1}^{r} L_{k} \cdot x_{\curlyvee j} k(1-R F)}{\sum_{j=1}^{r} \sum_{k=1}^{r} L_{k} \cdot x_{l j k}\left(T R_{\backslash j}+O P_{\backslash k}\right)+\sum_{j=1}^{r} \sum_{k=1}^{r} L_{k} \cdot x_{\curlyvee j k}\left[\left(T R_{r_{j} k}+O P_{\curlyvee k}\right)+R F\left(R T R_{k}+O P_{\backslash k}\right)-R E_{k}\right]}$ 


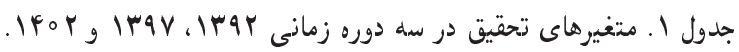

\begin{tabular}{|c|c|c|c|c|c|}
\hline Ifor & متغيرها & Iraf & متغيرها & Irar & متغيرها \\
\hline rIrs,949 & TRMr & $v \backslash r, r q \Delta r$ & TR $\| r$ & TYD & TR 11 \\
\hline $1998, \mathrm{VFT}$ & TRIYr & $G V 0, G \Delta Q V$ & TRIYr & rro & $T R \backslash Y \backslash$ \\
\hline$\| \wedge r T / 1 r$ & TRITr & GIr,rrAv & TRIrr & rlo & TRIr \\
\hline $1041, v \vee q$ & TRYIr & $r F q, q \circ V \wedge$ & TRYIr & Iro & TRYII \\
\hline $1 . \wedge \Delta, 1 \wedge \vee$ & TRYrT & $r G \varphi, \varphi \wedge V r$ & TRrrr & iro & TRYY \\
\hline$\| r \Lambda, \Delta q r$ & TRrrr & $r v q, 0991$ & TRrrr & ir。 & TRYrI \\
\hline FVV, YARI & OPIr & $190, r V F Y$ & OPIr & $\Delta \Delta$ & OPII \\
\hline 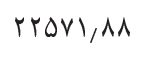 & OPrr & $V \Delta \Lambda 1, r r V$ & OPYY & rq०。 & OPYI \\
\hline$\Delta \Delta r \backslash, F r q$ & OP*rr & $M \Delta O F, \Delta I Y$ & OP*rr & srs & $\mathrm{OP}^{*} \mathrm{Y}$ \\
\hline 0,1 & $\mathrm{RF}$ & 0,1 & $\mathrm{RF}$ & 0,1 & $\mathrm{RF}$ \\
\hline 0,010 & $\mathrm{RF}^{*}$ & 0,010 & $\mathrm{RF}^{*}$ & 0,010 & $\mathrm{RF}^{*}$ \\
\hline$\wedge \& \wedge, \mid \uparrow q \mu$ & RTRr & $r q 1,0 \wedge q q$ & RTRr & 100 & RTRI \\
\hline$\wedge \& \wedge, \| \notin q \mu$ & $\mathrm{RTR}^{* r}$ & $r q 1,0 \wedge q q$ & $\mathrm{RTR}^{* r}$ & 100 & $\mathrm{RTR}^{*} \mathbf{I}$ \\
\hline$r G \circ \varphi \varphi, \varphi \wedge$ & REr & $\Lambda \vee \& V, q q Q$ & REr & roo & $\mathrm{REI}$ \\
\hline
\end{tabular}

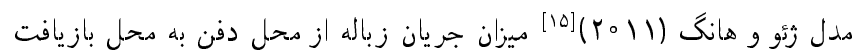

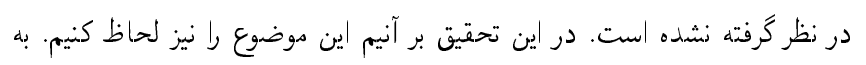
اين دليل متغيرهاى

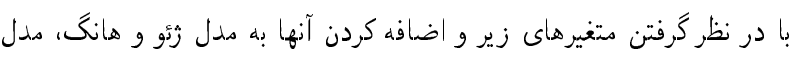
شدند. برنامه ريزى خطى كسرى تحقيق عبارت خواهد بود اضن ازئ $\operatorname{Pr}\left[\sum_{j=1}^{r} \sum_{k=1}^{r} L_{k}\left(x_{\backslash_{j k}}\left(1-R F^{*}\right)+R F . x_{r_{j k}}\right) \leq C L_{(t)}\right]$ $\geq 1-P_{c l}$ $\operatorname{Pr}\left[\sum_{j=1}^{r}\left(x_{r j k}+R F^{*} \cdot x_{\backslash j k}\right) \leq C F_{(t)}\right] \geq 1-P_{k C F} k=1, r, r$ $\operatorname{Pr}\left[\sum_{i=1}^{r} x_{i j k} \geq W G_{j k}(t)\right] \geq 1-P_{j k, W G} \quad j=1, r, r$ $k=1, r, \Gamma \quad x_{i j k} \geq 0 i=1, \Gamma \quad j=1, \Gamma, \Gamma \quad k=1, \Gamma, \Gamma$

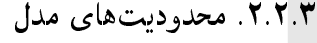

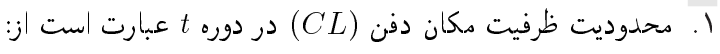
$\operatorname{Pr}\left[\sum_{j=1}^{r} \sum_{k=1}^{r} L_{k}\left(x_{\backslash j k}+R F \cdot x_{r_{j k}}\right) \leq C L_{(t)}\right] \geq 1-P_{c l}$ r. محدوديت ظرفيت تأسيسات بازيافت (CF) در دوره t مطابق فرمول If 1 در

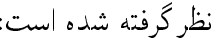

$\operatorname{Pr}\left[\sum_{j=1}^{r} x_{r_{j k}} \leq C F_{(t)}\right] \geq 1-P_{k C F} \quad K=1, r, r$ r. محدوديت تقاضاى مديريت ضايعات (WG) در منطقهى ز در دوره k جنين

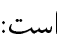
$\operatorname{Pr}\left[\sum_{i=1}^{r} x_{i j k} \geq W G_{j k}(t)\right] \geq 1-P_{j k, W G} \quad j=1, r, r$ $k=1, r, r$

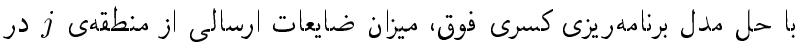

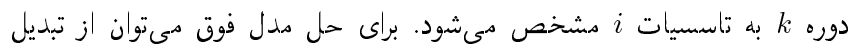

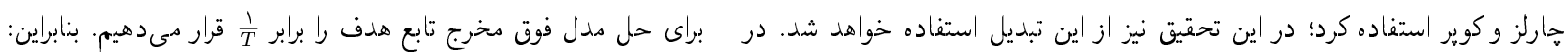
$\operatorname{Max} f=\frac{\sum_{j=1}^{r} \sum_{k=1}^{r} L_{k} \cdot x_{\curlyvee j k}(1-R F)+\sum_{j=1}^{r} \sum_{k=1}^{r} L_{k} \cdot R F^{*} \cdot x_{\backslash j k}}{\sum_{j=1}^{r} \sum_{k=1}^{r} L_{k} \cdot x_{\backslash j k}\left[\left(T R_{\backslash j k}+O P_{\backslash k}\right)+R F^{*}\left(R T R_{k}^{*}+O P_{\curlyvee k}^{*}+O P_{\curlyvee k}\right)-R E_{k}\right]+\sum_{j=1}^{r} \sum_{k=1}^{r} L_{k} \cdot x_{\curlyvee j k}\left[\left(T R_{\curlyvee j k}+O P_{\curlyvee k}+O P_{\curlyvee k}^{*}\right)+R F\left(R T R_{k}+O P_{\backslash k}\right)-R E_{k}\right]}$ 
لِّ از حل مدل فوق در سطوح احتمال مختلف، دقادير متغير تصميم را با استفاده از فرمول

\section{T.Y.r. مدل CCP با هدف كمينdسازى هزينه}

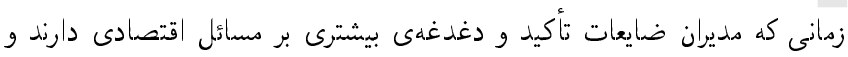

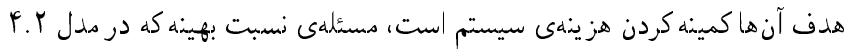

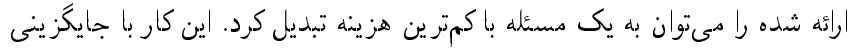

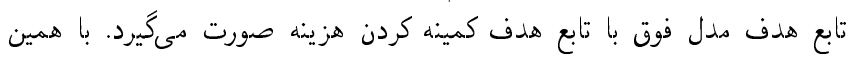

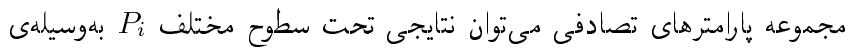

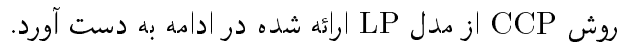
$\operatorname{Min}=\sum_{j=1}^{r} \sum_{k=1}^{r} L_{k} \cdot x_{\backslash j k}\left[\left(T R_{\backslash_{j k}}+O P_{\backslash k}\right)+R F^{*}\left(R T R_{k}^{*}\right.\right.$ $\left.\left.+O P_{r k}^{*}+O P_{r k}\right)-R E_{k}\right]+\sum_{j=1}^{r} \sum_{k=1}^{r} L_{k} \cdot x_{\curlyvee j k}\left[\left(T R_{r j k}\right.\right.$

$\left.\left.O p_{\ulcorner k}+O P_{\ulcorner k}^{*}\right)+R F\left(R T R_{k}+O P_{\backslash k}\right)-R E_{k}\right]$

$\operatorname{Pr}\left[\sum_{j=1}^{r} \sum_{k=1}^{r} L_{k}\left(x_{\backslash j k}\left(1-R F^{*}\right)+R F \cdot x_{\Upsilon_{j k}}\right) \leq C L_{(t)}\right]$

$($ (r०)

$$
\geq 1-P_{c l}
$$

$\operatorname{Pr}\left[\sum_{j=1}^{r}\left(x_{\curlyvee j k}+R F^{*} \cdot x_{\backslash j k}\right) \leq C F_{(t)}\right] \geq 1-P_{k C F} \quad k=1, r, r$ $\operatorname{Pr}\left[\sum_{i=1}^{r} x_{i j k} \geq W G_{j k}(t)\right] \geq 1-P_{j k, W G} \quad j=1, r, \Gamma$

$$
k=1, r, r \quad x_{i j k} \geq \circ \quad i=1, r \quad j=1, r, r \quad k=1, r, r
$$

هدف تحقيق حاضر تعيين ميزان ضايعات ارسالى از منطقهى i به تأسيسات ز

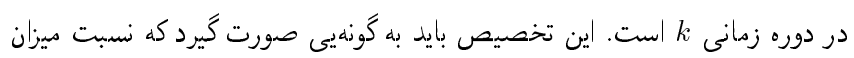

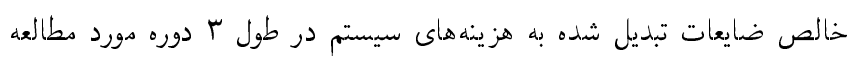

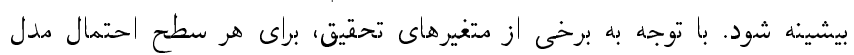

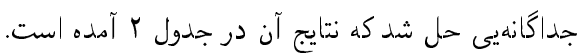

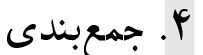

در اين يروهش، هدف تعيين ميزان ضايعات ارسالى از ب منطقهى شهر يزد به دو دحل

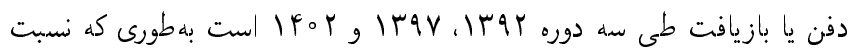

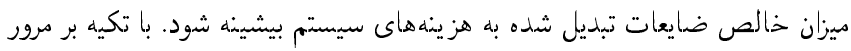

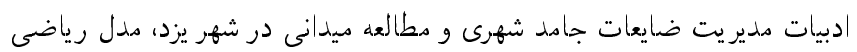

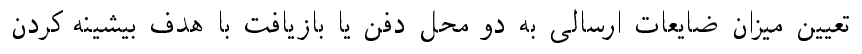

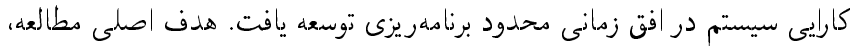

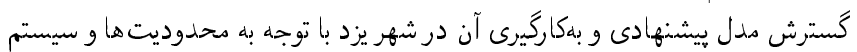

$\sum_{j=1}^{r} \sum_{k=1}^{r} L_{k} \cdot x_{\backslash^{j k}}\left[\left(T R_{\backslash_{j k}}+O P_{\backslash_{k}}\right)+R F^{*}\left(R T R_{k}^{*}\right.\right.$

$$
\begin{aligned}
& \left.\left.+O P_{r \nmid}^{*}+O P_{\curlyvee k}-R E_{k}\right)\right]+\sum_{j=1}^{r} \sum_{k=1}^{r} L_{k} \cdot x_{\curlyvee j k} \\
& {\left[\left(T R_{r_{j k}}+O P_{r_{k}}+O P_{\ulcorner k}^{*}\right)+R F\left(T R R_{k}+O P_{\backslash k}\right)\right.} \\
& \left.-R E_{k}\right]=\frac{1}{T}
\end{aligned}
$$

$\sum_{j=1}^{r} \sum_{k=1}^{r} L_{k} \cdot T \cdot x_{\backslash_{j k}}\left[\left(T R_{\backslash_{j k}}+O P_{\backslash_{k}}\right)+R F^{*}\left(R T R_{k}^{*}\right.\right.$

$$
\begin{aligned}
& \left.\left.+O P_{\curlyvee \uparrow}^{*}+O P_{\curlyvee k}-R E_{k}\right)\right]+\sum_{j=1}^{r} \sum_{k=1}^{r} L_{k} \cdot T \cdot x_{\curlyvee j k} \\
& {\left[\left(T R_{r^{\prime} k}+O P_{\curlyvee k}+O P_{\ulcorner k}^{*}\right)+R F\left(T R R_{k}+O P_{\backslash k}\right)\right.} \\
& \left.-R E_{k}\right]=1
\end{aligned}
$$

در نتيجه شكل تابع هدف عبارت خواهد بود از:

$$
\begin{aligned}
\operatorname{Max} & f=\frac{\sum_{j=1}^{r} \sum_{k=1}^{r} L_{k} \cdot x_{\curlyvee j k}(1-R F)+\sum_{j=1}^{r} \sum_{k=1}^{r} L_{k} \cdot R F^{*} \cdot x_{\backslash_{j k}}}{\frac{1}{T}} \\
= & \sum_{j=1}^{r} \sum_{k=1}^{r} L_{k} \cdot T . . x_{\ulcorner j k}(1-R F)+\sum_{j=1}^{r} \sum_{k=1}^{r} L_{k} \cdot R F^{*} \cdot T \cdot x_{\backslash j k}
\end{aligned}
$$

حال از تغيير متغير استفاده مىكنيم، بهطورى كه:

$y_{i j k}=T \cdot x_{i j k} \Rightarrow x_{i j k}=\frac{y_{i j k}}{T}$

با اعمال تغيير متغير فوق، مدل برنامهريزى خطى كسرى تحقيق به مدل برنادهريزى خطى زير تبديل خواهد شد. $\operatorname{Max} f: \sum_{j=1}^{r} \sum_{k=1}^{r} L_{k} . Y_{r j k}(1-R F)+\sum_{j=1}^{r} \sum_{k=1}^{r} L_{k} . Y_{\backslash_{j k}} . R F^{*}$

s.t:

$\sum_{j=1}^{r} \sum_{k=1}^{r} L_{k} . Y_{\backslash_{j k}}\left[\left(T R_{\backslash_{j k}}+O P_{\backslash k}\right)+R F^{*}\left(R T R_{k}^{*}+O P_{r \digamma}^{*}\right.\right.$

$$
\begin{aligned}
& \left.\left.+O P_{\curlyvee k}-R E_{k}\right)\right]+\sum_{j=1}^{r} \sum_{k=1}^{r} L_{k} Y_{Y j k}\left[\left(T R_{r_{j k}}+O P_{\curlyvee k}\right.\right. \\
& \left.\left.+O P_{\curlyvee k}^{*}\right)+R F\left(T R R_{k}+O P_{\backslash k}\right)-R E_{k}\right]=1
\end{aligned}
$$

$\left.\sum_{j=1}^{r} \sum_{k=1}^{r} L_{k} \cdot\left[y_{\backslash_{j k}}\left(1-R F^{*}\right)+y_{r j k} \cdot R F\right)\right] \leq T . C L_{(t)}$

$\sum_{j}\left(y_{\ulcorner j k}+y_{\curlyvee j k} \cdot R F^{*}\right) \leq T . C F_{(t)} \quad k=1, r, r$

$\sum_{i} y_{i j k} \geq T . W G_{j k} \quad j=1, r, r \quad k=1, r, \Gamma$

$y_{i j k} \geq \circ \quad i=1, r \quad j=1, r, r \quad k=1, r, r$ 
جدول r. مقدار ضايعات ارسالى از منطقهى i به تأسيسات j در دوره زمانى k.

\begin{tabular}{|c|c|c|c|c|c|c|c|c|c|}
\hline 0,90 & 0,9 & $0, v \Delta$ & 0,0 & $0, r \Delta$ & 0,1 & 0,00 & 0,01 & $\circ$ & $p$ \\
\hline 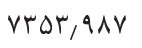 & IrV,VqAr & GrAT, rGA & $v r \Delta r, q \wedge v$ & Irv, vqAr & GYAr, rGA & $\Delta \Delta F r, 011$ & $r \wedge \circ 1, v \Delta r$ & YIrV, VAS & XYII \\
\hline $109 \Delta r, 9 r$ & $\| f \wedge \vee v, \Delta \Delta$ & IrDGY,Or & $109 \Delta r, 9 r$ & $\| f \wedge \vee \vee, \Delta \Delta$ & 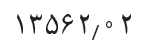 & $|r| \mid v, 0 q$ & logVr,IV & $q r \Delta G, g r \Lambda$ & XYIY \\
\hline $1 \wedge r \circ r, \circ q$ & $|V r 00, r|$ & $10 v v 0,8$ & $\mid A r \circ r, \circ q$ & $\left|v r_{0} 0, r\right|$ & $10 v v 0,8$ & $\mid r \circ q \circ, f r$ & $|r F| 0, r y$ & $101 \Lambda \circ, \Delta r$ & XYIr \\
\hline 。 & 。 & 。 & 。 & 。 & 。 & 。 & 。 & 。 & XYYI \\
\hline$\circ$ & 。 & $\circ$ & 。 & $\circ$ & $\circ$ & $\circ$ & $\circ$ & $\circ$ & XYYY \\
\hline 。 & 。 & $\circ$ & 。 & $\circ$ & $\circ$ & 。 & 。 & 。 & XrYr \\
\hline$\circ$ & $\circ$ & $\circ$ & $\circ$ & $\circ$ & $\circ$ & $\circ$ & $\circ$ & $\circ$ & XYMI \\
\hline$\circ$ & 。 & 。 & 。 & 。 & 。 & 。 & $\circ$ & 。 & XYTY \\
\hline 。 & $\circ$ & $\circ$ & $\circ$ & $\circ$ & $\circ$ & $\circ$ & 。 & 。 & Xrrr \\
\hline AVREY, YY & $q \backslash \vee \Delta \Lambda, \Delta \Lambda$ & $\Lambda 1 \circ \circ \Delta, \Delta v$ & AVTGY, rr & $q \backslash \vee \Delta \Lambda, \Delta \Lambda$ & $\Lambda 1 \circ \circ \Delta, \Delta v$ & VGGAr, 99 & VYTGY, & GAYTV,Ar & $x 111$ \\
\hline$\Lambda G r V F, \Delta r$ & $\Lambda F Y \backslash \Lambda, T F$ & $\Lambda \circ \Delta G \Psi, \Lambda \Gamma$ & $\Lambda G T V Y, \Delta r$ & $\Lambda F Y \backslash \Lambda, r F$ & $\Lambda \circ \Delta G \Psi, \Lambda r$ & VGOD $1,9 r$ & vrorq,or & $\Leftrightarrow \wedge \wedge \wedge \Delta, \uparrow \wedge$ & $X \| Y$ \\
\hline $1004 r q, 8$ & $q \vee q r r, 14$ & $q \mu G \wedge r, r F$ & $100 \% r q, 8$ & $q \vee q r r, 14$ & $q r G \wedge r, r r$ & $\wedge 9 \circ 1 \varepsilon, \Delta \Lambda$ & $\Lambda F r q q, \wedge \Delta$ & 10101,04 & $x \| r$ \\
\hline $9 \Delta \circ \circ \varepsilon, \Delta r$ & QTYVT,OV & AVGYI, MI & $9 \Delta \circ \circ 9, \Delta r$ & QRTVT,OV & AVGY,$M I$ & $\Lambda r \Delta \Delta F, 01$ & VVY\&9,99 & VYATY,QG & $X|r|$ \\
\hline $10 r F \Delta \circ, r$ & $99 \Delta \circ r, q \varphi$ & $q r \Delta \circ V, q \varphi$ & $10 r y \Delta 0, r$ & $99 \Delta \circ r, q \varphi$ & $q r \Delta \circ v, q r$ & $\Lambda q \circ r r$ & Arorg, of & $V \wedge \Delta Y \backslash, T V$ & XIYY \\
\hline 119141,4 & $110 v \circ r, q$ & 109199,1 & $1191 \pi 1,4$ & $11 \Delta v \circ r, q$ & 10919991 & loroIV & $q v \mid r v, q r$ & $q 1 r r_{0}, 1 r$ & XIYr \\
\hline$V \wedge q q_{0}, r q$ & $V G V T_{0}, \mathcal{G V}$ & $V r q \Delta q, r r$ & $V \Delta q F_{0}, Y Y$ & $V G V Y \circ, P V$ & Vrq0Q, rr & $G \wedge \wedge Y \wedge, 01$ & $94994, V 9$ & $q \circ q r \Delta, \Delta r$ & XITI \\
\hline$\Lambda \Delta I T Y, r \Lambda$ & ArVro, vr & VA\&VY,AY & $\Lambda \Delta I Y Y, r \Lambda$ & ArVTO,Vr & VA\&VY,Ar & VErro, 01 & GqVGO,IV & $q \Delta v \circ q, r q$ & XITY \\
\hline$q \wedge q \wedge \Delta, 4 q$ & 98901,99 & $q 1 \uparrow \wedge \Delta, \uparrow \Delta$ & $q \wedge q \wedge \Delta, 4 q$ & $98 Y 01,99$ & $91 \uparrow \wedge \Delta, 4 \Delta$ & $\Lambda \notin \mu \circ 0,01$ & $A M Y F, \Delta V$ & $V G Y \circ \Lambda, \circ \Delta$ & XIrr \\
\hline$r / \wedge r E+\circ V$ & $V, f \Delta \mathrm{E}+\circ \vee$ & $V, \& Y E+\circ \vee$ & $r / \wedge r E+\circ \vee$ & $V, f \Delta E+\circ V$ & $V, \& Y E+\circ V$ & $1, \circ 9 \mathrm{E}+\circ \wedge$ & $1, \uparrow r E+\circ \Lambda$ & $1, \mathrm{VrE}+\circ \wedge$ & هزينه \\
\hline $1, \nabla \vee E-\circ r$ & $0, \Delta q E-\circ \varphi$ & $\Delta, V \varphi E-\circ \varphi$ & $1, \forall V E-\circ r$ & $0, \Delta 9 E-\circ \varphi$ & $\Delta, v \varepsilon E-\circ \varphi$ & $r, q \varphi \mathrm{E}-\circ \varphi$ & $r, \Delta \circ E-\circ \varphi$ & $1, \wedge \Delta \mathrm{E}-\circ f$ & كارايى \\
\hline
\end{tabular}

در سطح P Pe Pادل با افزايش در سطح ريسك تخطى دعادلات هحدودكندهى

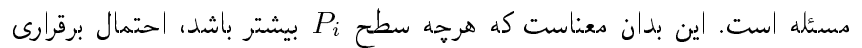

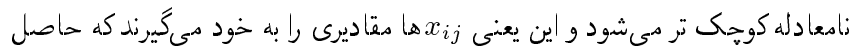
D Eaij xij

$p\left(\sum \sum a i j x i j \leq B\right) \geq 1-P_{i}$

هرجه مقدار Pi افزايش مىيابد سطح كارايى (يعنى هزينهى كمتر و مقدار

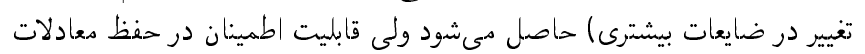

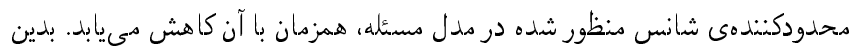
زترتيب مىتوان گفت تصميمهايى كه با سطح هايين

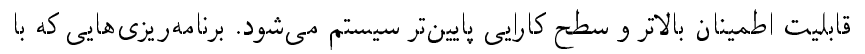
توجه به سطح بالاى

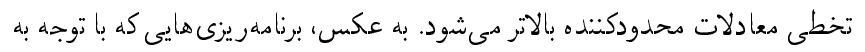

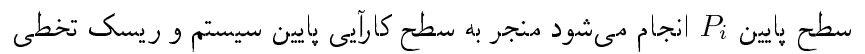

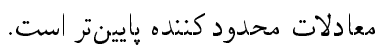
براى مثال زمانى كه 4 F r نشان داده شده است كه يك سطح بيشتر

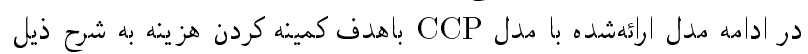

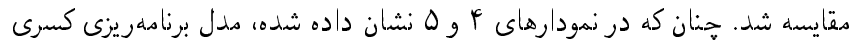

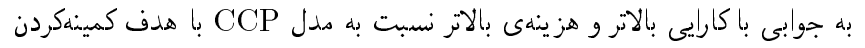

دفع زبالههاى جاهد شهرى آن است تا بهعنوان راهنمايى براى تصميميرندكان در

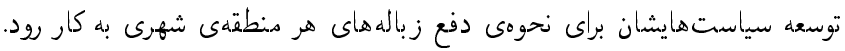

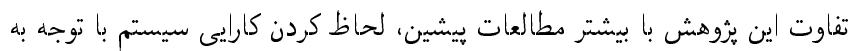

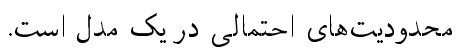

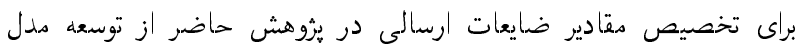

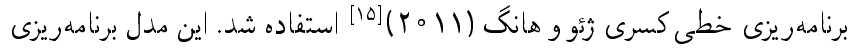

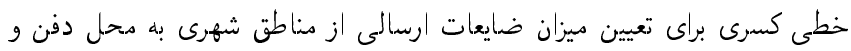

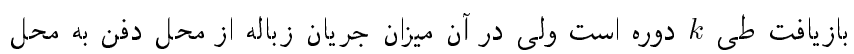

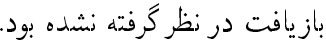

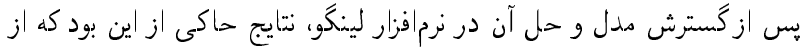

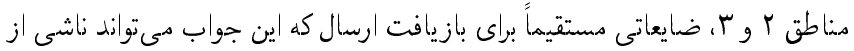

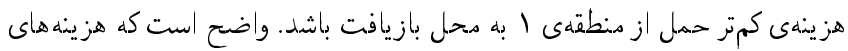

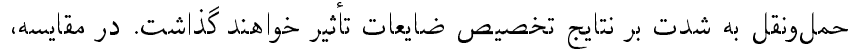

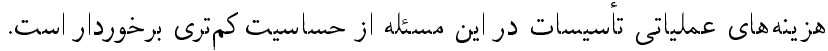
نتيجه دحاسبات نشان مى دهد كه سطح

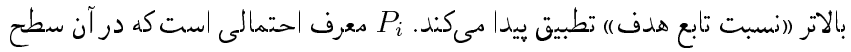

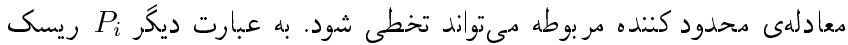

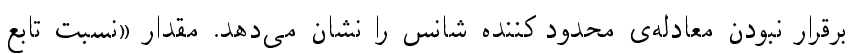

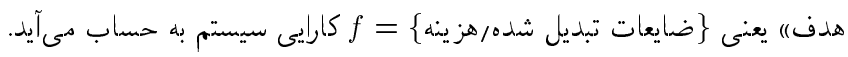

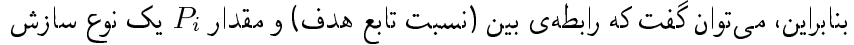
بين كارايى سيستم و ريسك تخطى دعادلهى دحدودكننده را نشان مى دهد. افزايش 


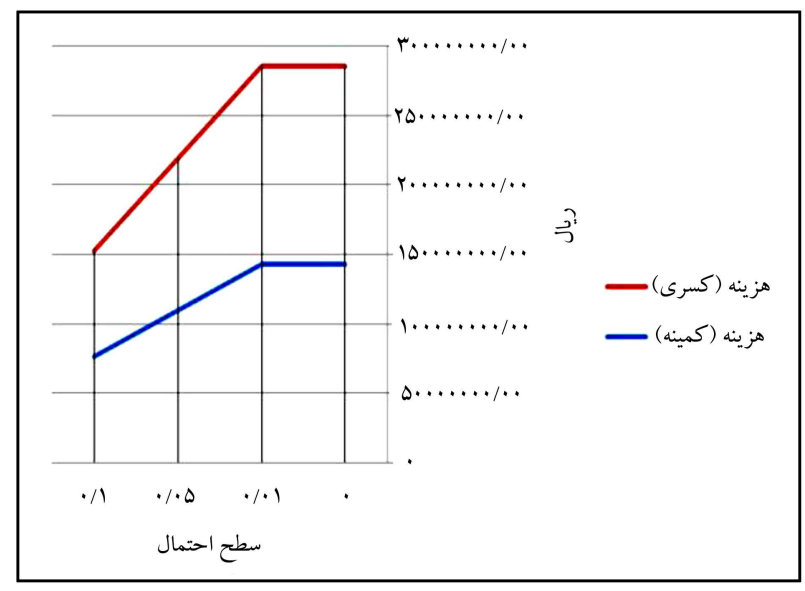

نمودار ه. مقايسهى هزينهى مدل برنامهر يزى كسرى با مدل CCP كمتر ين هزينه.

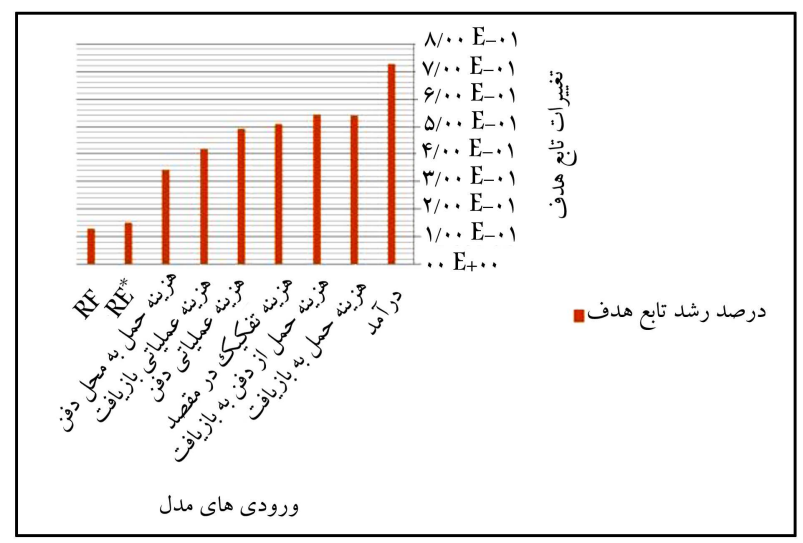

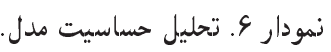

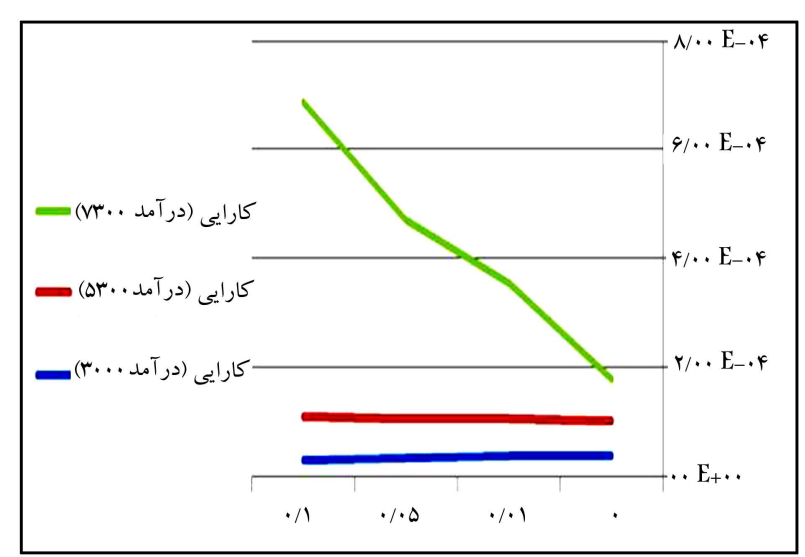

نمودار V. مقايسهى كاريى مدل برنامهر يزى كسرى با تغيير درآمد.

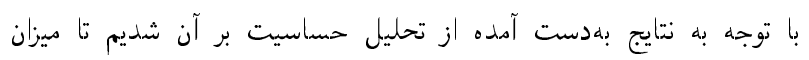

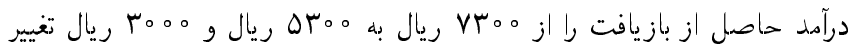

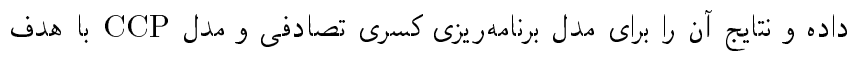

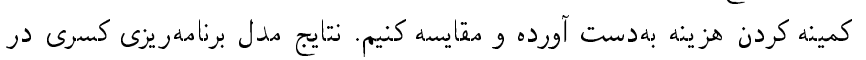

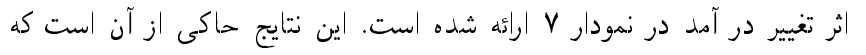

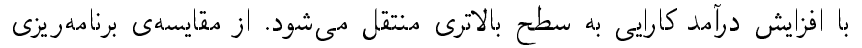
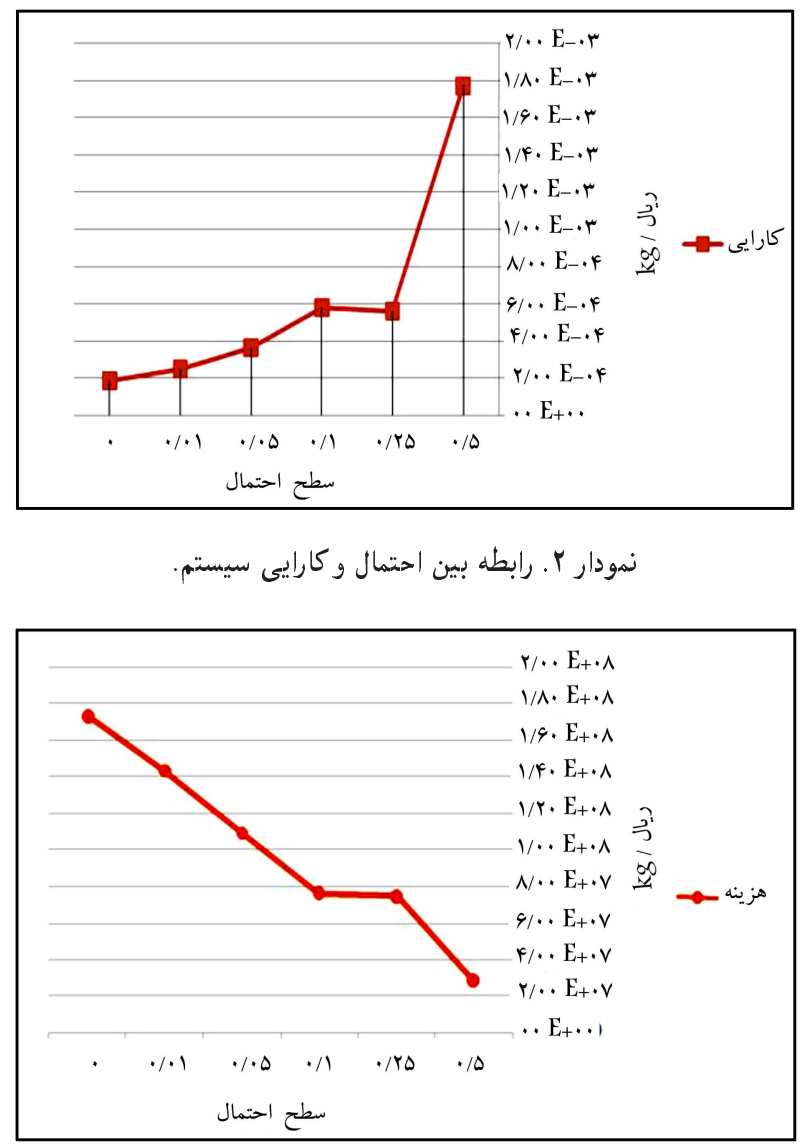

نودار با. رابطه بين احتمال و هزينهى سيستم.

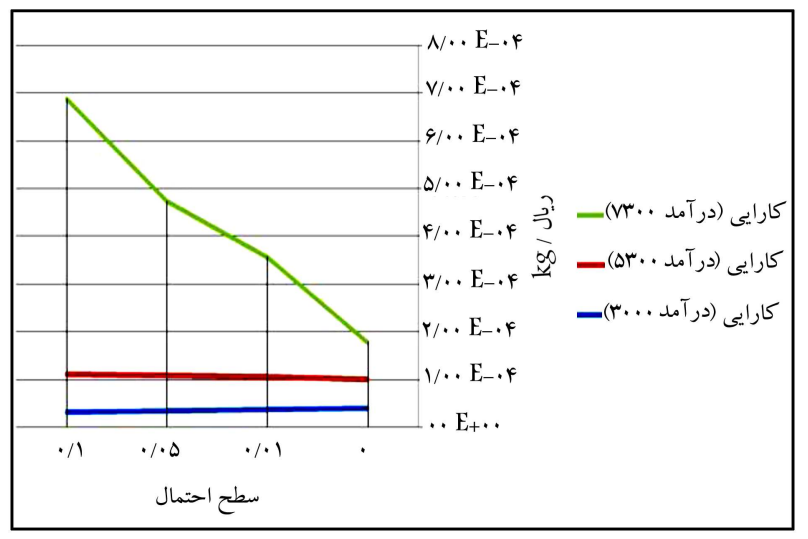

نمودار Fا. مقايسهى كارايى مدل برنامهريزى كسرى با مدل CCP كمترين هزينه.

هزينه منجر مى شود. بنابراين جنانجه هدف از يباده كردن مدل رسيدن به كارايى بالاتر

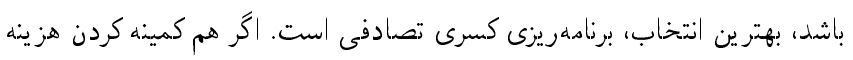

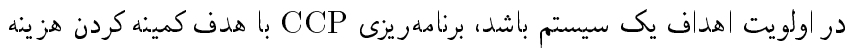

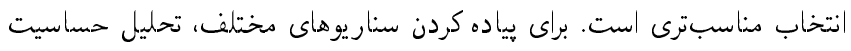

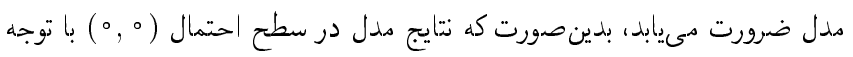

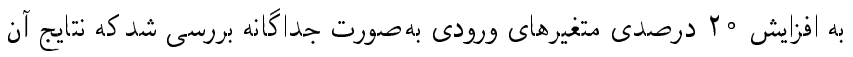

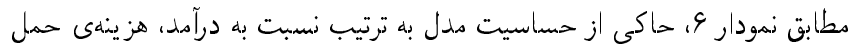

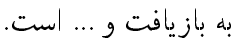




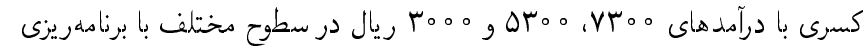

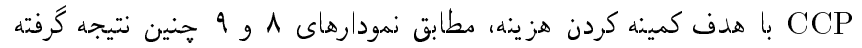

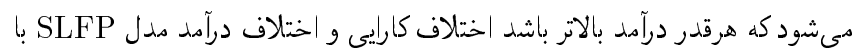

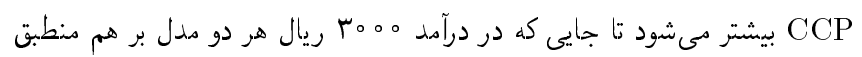
مى شود.

\section{ه. نتيجلةكيرى}

در اين تحقيق سعى بر آن است تا كارايى سيستم مديريت ضايعات بـات بيشينه

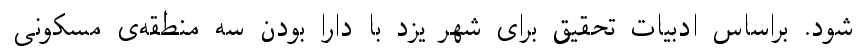

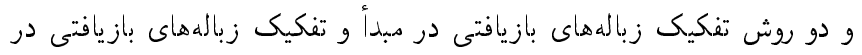

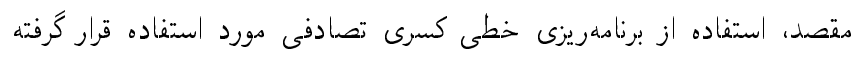

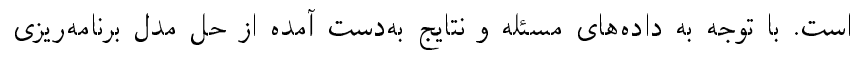

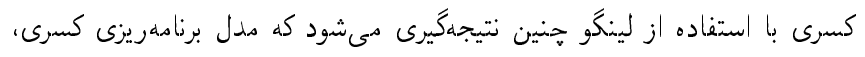

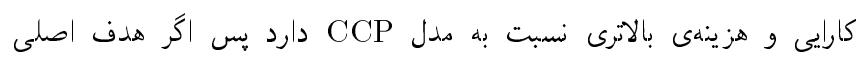

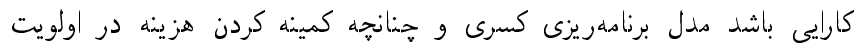

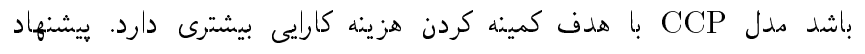

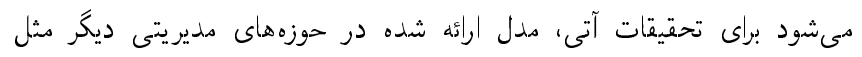

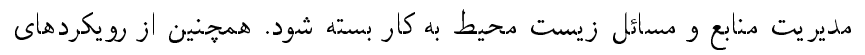
برنامهريزى اعداد صحيح، مدلسازى خطى كسرى و بهينه بهازى استوار استفاده شود.

يانوشتها

1. solid waste

2. Tanskanen

3. Macdonald

4. Englehardt

5. Chang and Wie

6. Petts

7. Shekdar \& et.al

8. Solano

9. Costi \& et.al

10. Hung \& et.al

11. Zhu \& et.al

12. Linear fractional programming

13. stochastic linear fractional programming

14. chance-constrained programming

\section{منابع (References)}

1. Omranei, Gh., and Nakhjavanei,A., Solid Waste (recycling), Volume III, Publishing Rafi thoughts. Tehran (2009).

2. Rafiei, R., Salman Mahinei, A. and khorasanei, N. "Life cycle environmental assessment of municipal waste management systems", Journal of Agricultural Sciences and Natural Resources, 16, pp.1-10 (2009)

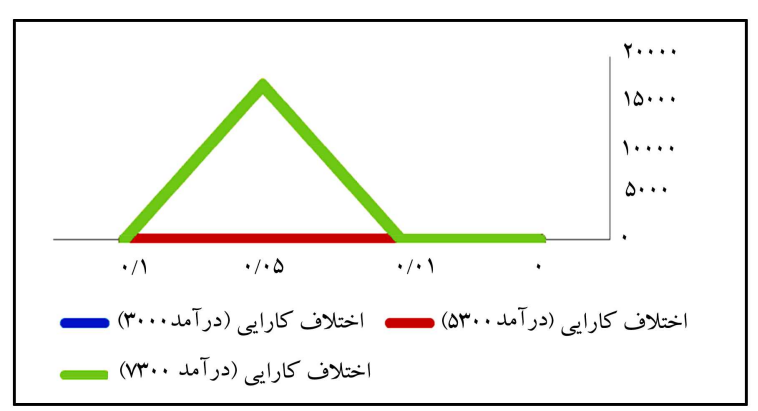

نمودار ^. مقايسهى اختلاف كارايى مدل برنامهر يزى كسرى با مدل CCP كمترين هزينه در درآمدهاى مختلف.

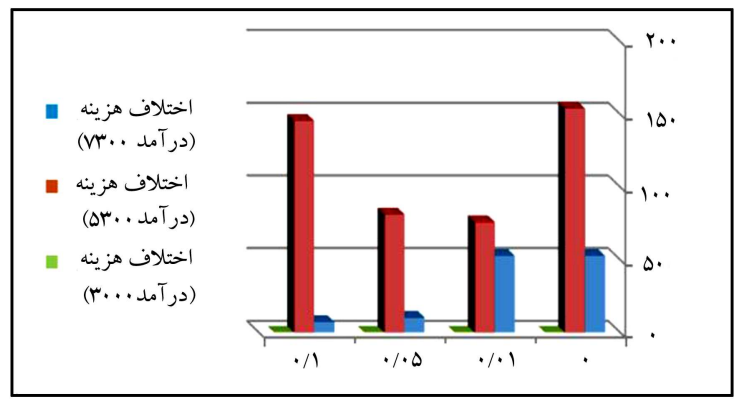

نمودار 9 ـ مقايسهى اختلاف هزينه مدل برنامهر يزى كسرى با مدل CCP كمترين هز ينه در درآمدهاى مختلف.

3. Morrissy, A.J. and Brown, J. "Waste management models and their application to sustainable waste management", Waste Management, 24, pp. 297-308 (2003).

4. Klundert, A. and Anschutz, J. "Integrated sustainable waste management - the concept tools for decision makers experiences from the urban waste expertise programme" Netkerlands (1995-2001).

5. Englehardt, J.D. and Lund, J.R. "Economic analysis of recycling for small municipal waste collectors", Journal of Resource Management and Technology, 18(2), pp. 8496 (1990).

6. Birge, J.R. "Solution methods for stochastic dynamic linear programming", Computers Operations Research (1980).

7. Tanskanen, J.H. "Strategic planning of municipal solid waste management", Resources, Conservation and Recycling, 30, pp. 111-133 (2000).

8. MacDonald, M.L. "A Multi-attribute spatial decision support system for solid waste planning", Comput. Environ. and Urban. Systems, 20(1), pp. 1-17 (1996b).

9. Chang, N.B. and Wei, Y.L. "Strategic planning of recycling drop- off? stations and collection network by multiobjective programming", Environmental Management, 24(2), pp. 247-263 (1999).

10. Petts, J. "Municipal waste management: inequities and the role of deliberation", Risk Analysis, 20(6), pp. 821$832(2000)$. 
11. Shekdar, A.V. and Mistry, P.B. "Evaluation of multifarious solid waste management systems-A goal programming approach", Waste Management and Research, 19, pp. 391-402 (2001).

12. Solano, E. Ranji Ranjithan, S. and Barlaz, Morton A. and Downey Brill, E. "Life- cycle-based solid waste management. I: Model development", Journal of Environmental Engineering, pp. 981-993 (2002).

13. Costi, P. Minciardi, R. Robba, M. Rovatti, M. and Sacile, R. "An environmentally sustainable decision model for urban solid waste management", Waste Management, 24, pp. 277-295 (2004).

14. Hung, M.L. Ma, H.W. and Yang, W.F. "A novel sustainable decision making model for municipal solid waste management", Waste Man agement, 27, pp. 209-219 (2007).

15. Zhu, H. and Hung, G.H. "SLFP: A stochastic linear fractional programming approach for sustainable waste management", Waste Management, 31, pp.2612-2619 (2011).

16. Charles, V, and Dutta, D. "Extremization of multiobjective stochastic fractional programming problem", Annals of Operations Research, 143, pp. 297-304 (2006a).

17. Zare Mehrjerdi, Y. and Daneshmand, A. "A linear approximation method for solving a special class of the chance constrained programming problem", European Journal of Operational Research, 80(1), pp. (1995).

18. Zare Mehrjerdi, T. "Solving fractional programming problem through fuzzy goal setting and approximation", Applied Soft Computing, 11(2), pp. (2011).

19. Zare Mehrjerdi, Y. "Fractional chance constrained programming: A fuzzy goal programming approach", Inter- national Journal of Industrial Engineering and Production Research, 25(3), pp. (2013).

20. Zare Mehrjerdi, Y. "A chance constrained goal programming model of stochastic vehicle routing problem", Phd Dissertation, Oklahoma State University, Oklahoma,U.S.A (1986).

21. Zare Mehrjerdi, Y. "Chance constrained Programming of manpower grading problem in production area", Master Thesis, St.Marys University. San Antionio Texas, U.S.A (1982).

22. Zare Mehrjerdi, Y., Stochastic Programming, Mehrjerd Press, Tehran, Iran (In Persian) (2014).

23. Chadha, S.S. and chadha, V. "linear fractional programming and duality", Cent.Eur. J.Opar. Res, 15, pp. 119125 (2007).

24. Akhavan Niyakii, S.T., Probability Theory and Its Applications. Tehran, Sharif University of Technology, Scientific Publications (2005).

25. Guo, P. Huang, G.H. and Li, Y.P. Interval stochastic quadratic approach programming for municipal solid waste management", J. Environ. Eng. Sci., 7, pp. 568579 (2008).

26. Guo, P. Huang, G.H. and He, L. "ISMISIP: An inexact stochastic mixed integer linear semi-infinite programming approach for solid waste management and planning under uncertainty", Stoch. Environ. Res. Risk. Assess., 22, pp. 759-775 (2008b).

27. Wallace, S.W. and William, T.Z., Applications of Stochastic Programming, SIAM (2005).

28. Mirhossaini, A. and Adib, M. "Linear fractional programming duality", Zanjan University, Faculty of Science, Department of Mathematics (2010). 